\title{
Effets contrastés des prélèvements de bois sur la végétation de forêt sèche en zone frontalière dominico-haïtienne : comment les interpréter?
}

\section{Thomas MaY}

Universidad Estatal Amazónica (UEA)

Via a Napo km $21 / 2$, paso lateral

Puyo, Pastaza

Équateur

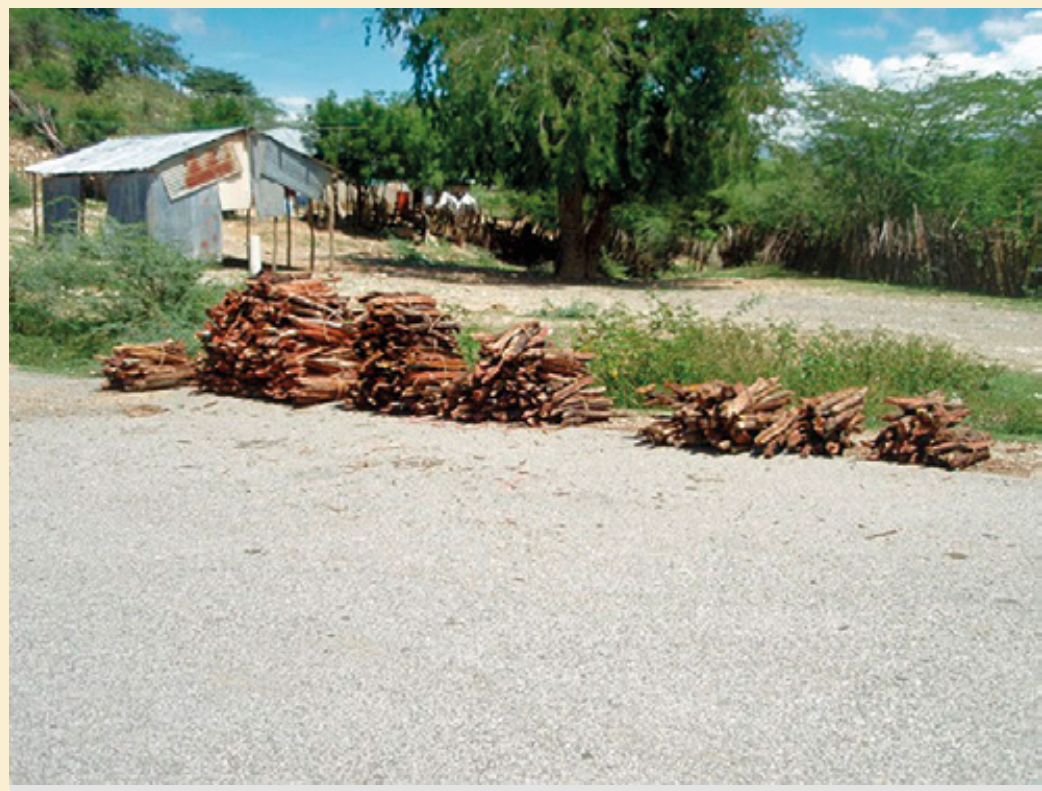

Photo 1.

Bois de feu à la vente au bord de la route (République dominicaine).

Dans certaines zones rurales, non seulement le charbon de bois

mais aussi le bois de feu font l'objet de commercialisation.

Photo T. May. 


\section{RÉSUMÉ}

\section{EFFETS CONTRASTÉS DES PRÉLĖVEMENTS DE BOIS SUR LA VÉGÉTATION DE FORÊT SĖCHE EN ZONE FRONTALIĖRE DOMINICO-HAÏTIENNE : COMMENT LES INTERPRÉTER ?}

En Haïti, le bois de feu et le charbon végétal sont des sources importantes d'énergie domestique, et le prélèvement de bois à des fins énergétiques représente un facteur de dégradation de la forêt sèche, en plus du parcours d'animaux domestiques (chèvres et vaches). En République dominicaine limitrophe, tandis qu'il y a trois décennies la situation était similaire, les conditions ont aujourd'hui changé et les forêts sèches montrent des signes de régénération. Dans l'extrême sud de la ligne de frontière entre les deux pays, près d'Anseà-Pitre et Pedernales, l'opportunité se présente de comparer l'état de la forêt sèche des deux côtés, dans des conditions géologiques et climatiques très semblables. Notre étude montre que le couvert des individus arbustifs et arborés et la hauteur des arbres sont plus élevés en République dominicaine, tandis que le nombre d'individus multicaules issus de régénération végétative est plus élevé en Haïti. En général, la composition spécifique est similaire des deux côtés de la frontière, mais des différences significatives apparaissent dans les fréquences et les valeurs d'abondance-dominance. Acacia scleroxylon, Amyris elemifera, Bursera simarouba, Capparis ferruginea et Guaiacum sanctum sont plus fréquents en République dominicaine, et Acacia macracantha, Senna atomaria, Phyllostylon brasiliense et les deux cactacées Pilosocereus polygonus et Opuntia sp. sont au contraire plus fréquents en Haïti. Ces différences sont imputables à l'autécologie des espèces (ex. : capacité de coloniser les terrains perturbés, capacité de régénération végétative) plutôt qu'à des préférences dans leur utilisation comme bois de feu ou de charbon.

Mots-clés : charbon végétal, impact de l'utilisation humaine, structure de la végétation, fréquence et abondance-dominance des espèces végétales, résilience, zone frontalière, République dominicaine, Haïti.

\section{ABSTRACT}

\section{INTERPRETING THE CONTRASTING AFFECTS OF WOOD-CUTTING ON DRY FOREST VEGETATION IN THE DOMINICAN REPUBLIC-HAITI BORDER AREA}

In Haiti, wood and charcoal are important sources of domestic fuel, and wood-cutting for fuel is a major cause of degradation of the region's dry forests, together with livestock pasturing (cattle and goats). In the Dominican Republic across the border, while the situation was similar some thirty years ago, conditions have changed and dry forests are showing signs of regeneration. The southernmost part of the border area between the two countries, around Anse-à-Pitre and Pedernales, offers an opportunity to compare the condition of dry forests on either side of the border, where the climatic and geological conditions are virtually identical. Our study shows denser tree and shrub cover and higher trees on the Dominican Republic side and a larger number of individual trees regenerating from multiple stems on the Haitian side. In general, the species composition is similar on both sides of the border, but there are significant differences in their frequency and abundance-dominance. Acacia scleroxylon, Amyris elemifera, Bursera simarouba, Capparis ferruginea and Guaiacum sanctum are more frequent in the Dominican Republic, while Acacia macracantha, Senna atomaria, Phyllostylon brasiliense and the two cacti Pilosocereus polygonus and Opuntia sp. are more frequent in Haiti. These differences may be attributed to the autecology of the species (e.g. capacity for colonising disturbed terrain and for vegetative regeneration) rather than to preferences for their use such as for firewood and charcoal.

Keywords: charcoal, impact of human uses, vegetation structure, frequency and abundance-dominance of plant species, resilience, border zone, Dominican Republic, Haiti.

\section{RESUMEN}

\section{¿CÓMO INTERPRETAR LOS DESIGUALES EFECTOS DE LA EXTRACCIÓN DE MADERA EN LA VEGETACIÓN DEL BOSQUE SECO DEL ÁREA FRONTERIZA DOMINICO-HAITIANA?}

La leña y el carbón vegetal constituyen importantes fuentes de energía doméstica en Haití. Su extracción con fines energéticos supone un factor de degradación del bosque seco que se añade al pastoreo de animales domésticos (cabras, chivos y vacas). En en las zonas limítrofes de la República Dominicana la situación era similar hace tres décadas, pero actualmente las condiciones han cambiado y los bosques secos muestran señales de regeneración. En el extremo sur de la línea fronteriza entre los dos países, cerca de Anse-à-Pitre y Pedernales, se presenta la ocasión de comparar el estado del bosque seco a ambos lados, en condiciones geológicas y climáticas muy parecidas. Nuestro estudio muestra que la cubierta arbustiva y arbórea y la altura de los árboles es superior en la República Dominicana, mientras que el número de individuos multicaules procedente de regeneración vegetativa es superior en Haití. En general, la composición específica es similar a ambos lados de la frontera, pero aparecen diferencias significativas en las frecuencias y los valores de abundancia-dominancia. Acacia scleroxylon, Amyris elemifera, Bursera simarouba, Capparis ferruginea y Guaiacum sanctum son más frecuentes en la República Dominicana y, por el contrario, Acacia macracantha, Senna atomaria, Phyllostylon brasiliense y las dos cactáceas Pilosocereus polygonus y Opuntia sp. son más frecuentes en Haití. Estas diferencias obedecen más a la autoecología de las especies (p. ej.: capacidad de colonizar suelos alterados y capacidad de regeneración vegetativa) que a preferencias en su uso para leña o carbón.

Palabras clave: carbón vegetal, impacto de la utilización humana, estructura de la vegetación, frecuencia y abundancia-dominancia de especies vegetales, resiliencia, área fronteriza, República Dominicana, Haití. 


\section{Introduction}

Pour des raisons climatiques, la productivité en biomasse des forêts sèches tropicales et subtropicales fluctue entre 8 et 21 tonnes par an et par hectare, ce qui correspond à 50 à $75 \%$ de la productivité des forêts humides (Murphy et Lugo, 1986). Selon les mêmes auteurs, la production de grume atteint deux tonnes au plus par hectare et par an. Malgré cette productivité relativement faible, un grand nombre de produits sont extraits de ces écosystèmes. Un des produits les plus importants est le bois énergie, tant comme bois de feu que comme bois transformé en charbon. Il faut y ajouter l'exploitation de bois pour des poteaux et le pâturage des ruminants (Murphy et Lugo, 1986), de même que la récolte de produits non ligneux.

En Haïti, pays le plus pauvre des deux sous-continents américains (Boccanfuso et Siméon, 2006), le bois de feu est fréquemment utilisé en milieu rural, mais le charbon de bois reste l'une des sources d'énergie les plus importantes en milieu urbain. Selon une étude récente, le seul bois de feu correspond à environ $55 \%$ de la consommation énergétique des ménages, et le charbon de bois en représente $17 \%$ (Racicot, 2011). En Haïti, l'efficience du processus de transformation du bois en charbon est basse puisque pour produire une tonne de charbon, selon la même étude, un équivalent de 5,6 tonnes de bois est utilisé. Bien que l'effet spécifique de l'extraction du bois de feu et du charbonnage sur l'écosystème n'ait pas été étudié en Haïti, des expériences et des analyses conduites en Afrique orientale (Hofstad, 1997 ; Tabuti et al., 2003 ; Mwampamba, 2007) et occidentale (Houtondji et al., 2013 ; Mama et al., 2014 ; Peltier et al., 2014) laissent supposer que les impacts négatifs de l'extraction du bois énergie sur la biodiversité et les ressources naturelles sont considérables en Haïti.

En République dominicaine, pays voisin d'Haïti, l'utilisation du bois de feu et du charbon de bois était encore très répandue dans les années 1980 (Hartshorn et al., 1981). Pendant les décennies suivantes, l'utilisation du gaz de cuisine, subventionné par les pouvoirs publics (Artana et al., 2006), a fortement augmenté en milieu urbain, et la demande en charbon de bois a considérablement diminué, de telle sorte que le bois énergie ne représentait plus que $12 \%$ de la consommation énergétique au début du siècle suivant (Díaz, 2003). Cela coïncide avec le constat qu'en beaucoup d'endroits de ce pays les forêts sèches ont amorcé un processus de régénération, comme cela a été notamment décrit pour l'île voisine de Puerto Rico (Molina Colón et Lugo, 2006) où ce processus avait commencé quelques décennies plus tôt. S'il demeure certain que d'autres facteurs ont également pu favoriser cette régénération, comme par exemple les alternatives d'activités pour la population rurale, la réduction du charbonnage a constitué un facteur déterminant. La coupe des arbres pour leur conversion en bois énergie représentait en effet la principale utilisation de ces écosystèmes (Hartshorn et al., 1981).

Dans l'extrême sud de la frontière dominico-haïtienne, près d'Anse-à-Pitre (Haïti) et Pedernales (République dominicaine), l’opportunité se présente de comparer directement

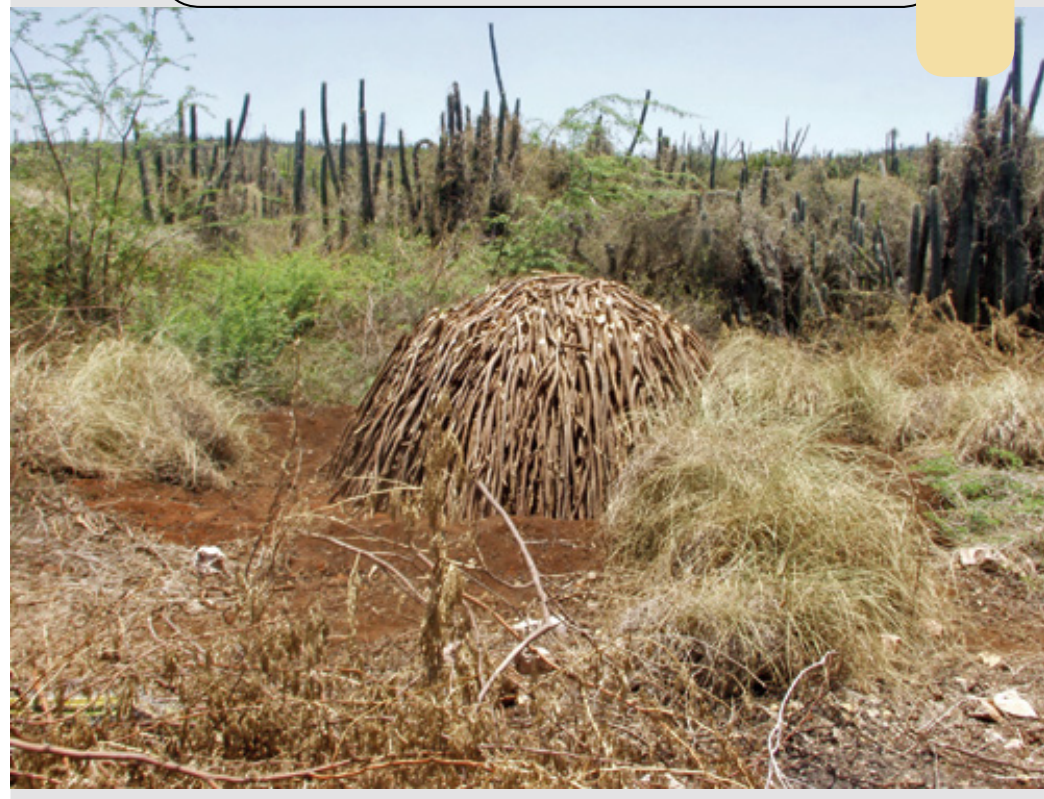

Photo 2.

Bois entassé pour produire du charbon dans un lieu d'exploitation récente, en forêt sèche près d'Anse-à-Pitre, Haïti. Les cactus dans le fond, non utilisables pour produire du charbon, sont épargnés.

Photo E. Rupp.

l'état de la végétation de la forêt sèche des deux côtés de la frontière en conditions climatiques, géologiques, topographiques et pédologiques similaires. À la suite de deux missions de reconnaissance réalisées à partir de novembre 2013, il a été confirmé que l'utilisation du bois pour produire du charbon est une activité répandue du côté haïtien, ce qui contraste avec la situation dominicaine où l'intensité de cette activité s'avère beaucoup plus réduite, voire absente. Les parcours de chèvres et vaches sont présents du côté haïtien, mais des incendies ou des traces d'incendies récents n'y ont pas été observés, au contraire d'autres sections de la zone frontalière dominico-haïtienne.

Ces informations recueillies sur le terrain, associées à des données bibliographiques, ont permis de formuler les hypothèses suivantes :

- l'utilisation forestière, principalement la coupe de bois pour la production de charbon, beaucoup plus intense du côté haïtien que du côté dominicain, a un impact sur la structure de la végétation dans la zone d'étude ;

- cette utilisation forestière a également un impact sur la fréquence, l'abondance et le couvert des différentes essences d'arbres et arbustes de la zone d'étude ;

- les peuplements des essences préférées comme bois énergie souffrent davantage que les autres essences de cet impact, même si les essences préférées comme bois de feu ou pour la fabrication de charbon sont plus fréquentes du côté de la République dominicaine.

Selon les résultats d'une étude réalisée en Haïti dans les années 1990 (Université de Louvain, 1998), les trois espèces Acacia macracantha, Prosopis juliflora et Senna atomaria sont les plus utilisées comme bois de feu ou pour la fabrication de charbon. Dans les trois cas, il s'agit d'espèces de la forêt sèche. Les trois sont présentes dans la zone d'étude bien que $P$. juliflora s'y rencontre seulement 

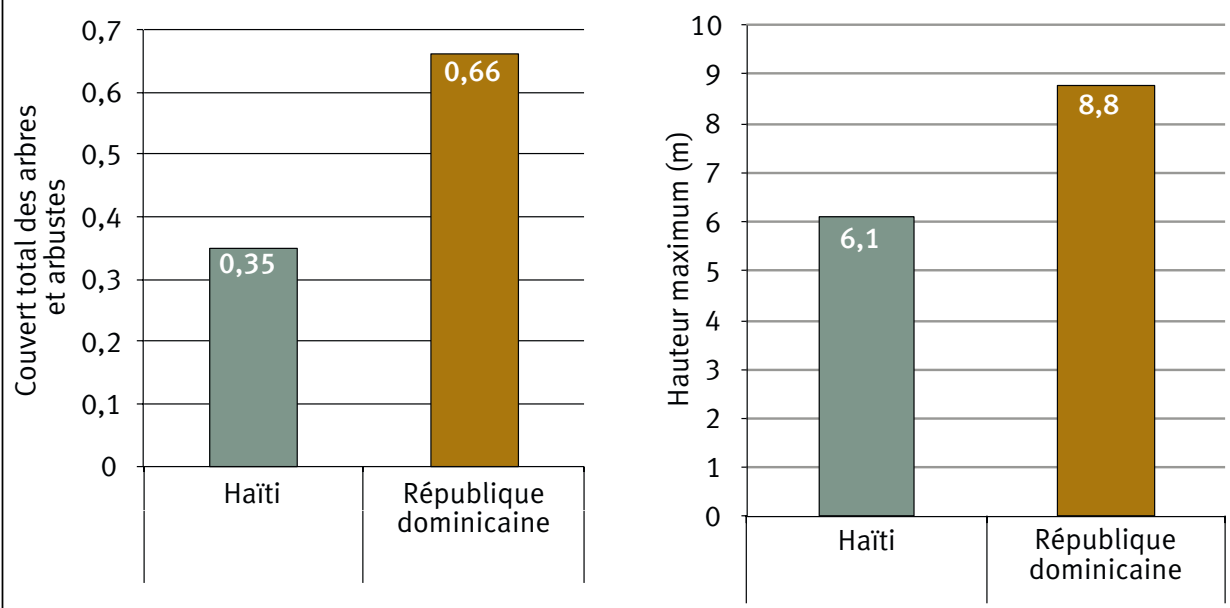

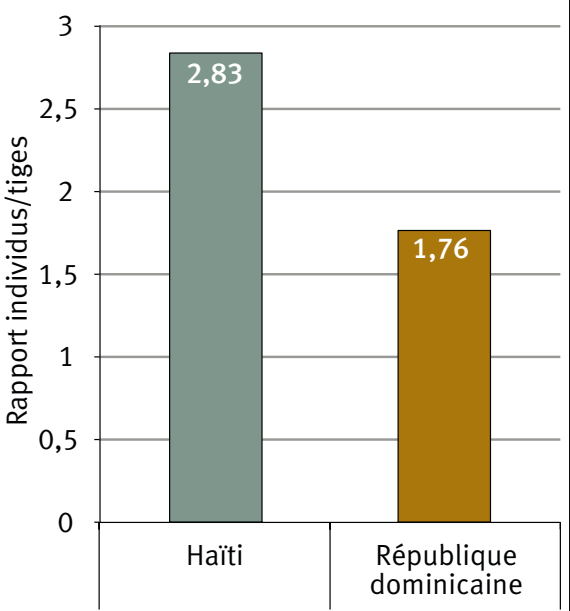

Figure 1.

Paramètres structuraux.

\section{Résultats}

\section{Paramètres structurels}

Pour chaque parcelle, les mesures des tiges ont été ventilées selon cinq classes de DHP $(<2 \mathrm{~cm}, 2-5 \mathrm{~cm}, 5-10 \mathrm{~cm}$, $10-20 \mathrm{~cm},>20 \mathrm{~cm}$ ) et cinq classes de hauteur ( $<0,5 \mathrm{~m}, 0,5-$ $1,5 \mathrm{~m}, 1,5-3 \mathrm{~m}, 3-5 \mathrm{~m},>5 \mathrm{~m}$ ). Pour chaque parcelle, la densité (nombre d'individus par hectare) a été calculée pour toutes les classes de diamètre et de hauteur, et les moyennes arithmétiques ont également été calculées. À l'aide du test $U$ de Mann-Whitney, il a été déterminé si les différences entre les moyennes des deux côtés de la frontière étaient statistiquement significatives ou non.

Les fréquences des espèces (nombre de relevés avec présence de l'espèce rapporté au total de relevés) ont été déterminées. Le test exact de Fisher, basé sur un tableau de contingence de $2 \times 2$ (présence/absence - Haïti/République dominicaine), a permis d'évaluer la significativité des différences observées entre les valeurs de fréquence des deux côtés de la frontière. Le test $U$ de Mann-Whitney a été utilisé pour déterminer si les différences entre les valeurs d'abondance-dominance (échelle de Braun-Blanquet) des deux côtés de la frontière étaient significatives. La valeur $\mathrm{P}=0,05 \mathrm{a}$ été adoptée comme limite de confiance. Le test $U$ a été choisi dans ce cas, de même que pour la comparaison des paramètres structuraux, parce qu'il est mieux adapté que les tests paramétriques pour analyser des données à distribution asymétrique (paramètres structurels) et des données à échelle ordinale (valeurs d'abondance-dominance selon BraunBlanquet). Le logiciel d'accès libre Vassarstats ${ }^{1}$ a été utilisé.

Les paramètres structurels (densité des tiges, densité des individus, rapport tiges/pied) dans les six relevés des endroits d'exploitation récente du côté haïtien ont été comparés avec les données des 18 relevées du côté haïtien, à l'aide des mêmes tests statistiques.
Dans les 18 parcelles situées en République dominicaine, la moyenne des valeurs du couvert total de la végétation d'une hauteur supérieure à $1 \mathrm{~m}$ était de $66 \%$, soit presque le double de la valeur moyenne de $35 \%$ obtenue dans les 18 parcelles localisées en Haïti (figure 1). De même, la moyenne de la hauteur maximum de la végétation ligneuse était nettement plus élevée en République dominicaine $(8,8 \mathrm{~m})$ qu'en Haïti $(6,1 \mathrm{~m})$, et la moyenne du rapport tiges/pied était supérieure en Haïti (2,83, pour 1,76 en République dominicaine). Pour les trois paramètres, les différences entre les valeurs des deux côtés de la frontière étaient significatives $(P=0,00$ pour le couvert total et pour le rapport tiges/pied; $P=0,01$ pour la hauteur maximum, selon le test $U$ de Mann-Whitney).

La densité moyenne des tiges (n/ha) pour les cinq classes de diamètre est représentée en haut de la figure 2. Les valeurs de densité étaient plus hautes pour les diamètres inférieurs à $10 \mathrm{~cm}$ du côté haïtien, et étaient plus élevées pour les diamètres supérieurs à $10 \mathrm{~cm}$ du côté dominicain. Selon le test $U$ de Mann-Whitney, ces différences étaient significatives pour les diamètres de 2 à $5 \mathrm{~cm}(P=0,02), 5$ à $10 \mathrm{~cm}$ $(P=0,05), 10$ à $20 \mathrm{~cm}(P=0,02)$ et plus de $20 \mathrm{~cm}(P<0,01)$, mais pas pour les diamètres de 0 à $2 \mathrm{~cm}$. Le bas de la figure 2 présente les densités des individus ( $\mathrm{n} / \mathrm{ha}$ ) pour les cinq classes de hauteur. Pour les parcelles de République dominicaine, la distribution des densités s'approchait d'une courbe en J inversé ; à l'exception de la classe de hauteur de 1,5-3 m qui représentait un faible maximum secondaire, la distribution pour les parcelles haïtiennes montrait un maximum absolu dans cette classe de hauteur. Pour les individus de la classe 0-0,5 m et de la classe > $5 \mathrm{~m}$, les densités étaient plus élevées en République dominicaine ; elles l'étaient en revanche en

${ }^{1}$ Cf. site www.vassarstats.org, visité en plusieurs occasions entre juin 2014 et mai 2015. 
Haïti pour les classes intermédiaires. Les différences étaient significatives pour la classe $0-0,5 \mathrm{~m}(\mathrm{P}=0,03)$ et pour la classe $5 \mathrm{~m}$ et plus $(\mathrm{P}<0,01)$, mais pas pour les classes intermédiaires.

Dans les six relevés montrant une exploitation récente de bois, tous situés du côté haïtien, pour la classe de diamètre inférieur à $2 \mathrm{~cm}$, les densités de tiges étaient plus élevées que dans les 18 parcelles sans exploitation récente de bois (figure 3), tandis que, pour toutes les autres classes de diamètre, les densités de tiges étaient moins élevées. Les différences étaient significatives seulement pour les classes de diamètre $5-10 \mathrm{~cm}(P=0,01$, test $U$ de Mann-Whitney). Les valeurs de la densité des individus étaient plus élevées dans les six parcelles à exploitation récente pour les deux classes de hauteur de 0-0,5 m et 0,5-1,5 m, au contraire des classes de hauteur au-dessus de 1,5 m (figure 3). Les différences étaient significatives seulement pour la classe de hauteur de $5 \mathrm{~m}$ et plus, selon le test $\mathrm{U}$ de Mann-Whitney.

\section{Composition}

Dans les 36 parcelles étudiées, 0,3\% des individus et $0,2 \%$ des tiges correspondaient aux six espèces ligneuses dont le genre et l'espèce n'ont pu être identifiés (tableau I). Pour ces plantes, les différences des fréquences et des valeurs d'abondance-dominance n'ont pas été analysées. La distribution de l'immense majorité des arbres et arbustes présents dans les relevés apparaît dans l'analyse suivante.

La plupart des espèces (19 sur 28 , soit $68 \%$ ) étaient présentes aussi bien dans les relevés du côté haïtien que dans ceux du côté dominicain. Les fréquences des arbres et arbustes Acacia macracantha, Senna angustisiliqua, Senna atomaria et de la liane Cissus trifoliata étaient plus élevées en Haïti, cela de manière significative (test exact de Fisher ; tableau I), tandis que les fréquences des cactacées Pilosocereus polygonus et Opuntia sp. mais aussi de l'arbre Phyllostylon brasiliense n'étaient que légèrement plus élevées en Haïti, les différences entre les valeurs n'étant cependant pas significatives (test exact de Fisher ; tableau I). Les fréquences des arbres et arbustes Acacia scleroxylon, Amyris balsamifera, Capparis ferruginea, Guaiacum sanctum et la succulente Agave antillarum étaient significativement plus élevées dans les relevées réalisés du côté dominicain (test exact de Fisher; tableau I). Dans les cas des arbres Bursera simaruba et Guaiacum officinale, les fréquences étaient aussi plus élevées du côté dominicain, mais pas de manière significative (test exact de Fisher, tableau I). Les fréquences des trois espèces de Croton étaient relativement basses et similaires dans les relevés des deux côtés de la frontière.
Figure 3.

Structure horizontale (en haut, tiges d'arbres et arbustes/hectare) et verticale (en bas, individus d'arbres et arbustes/hectare) dans des endroits avec et sans coupe récente du côté haïtien. 
Les valeurs d'abondance-dominance selon l'échelle de Braun-Blanquet montrent une distribution similaire. Les seules différences observées par rapport aux différences des fréquences sont que les valeurs d'abondance-dominance de Bursera simaruba sont significativement plus hautes du côté dominicain (test U de Mann-Whitney, tableau I), et que les différences des valeurs d'abondance-dominance ne sont pas significatives pour Cissus trifoliata.

Dans les six parcelles d'exploitation récente, les espèces à fréquence plus élevée étaient Phyllostylon brasiliense (photo 5) dont des nouveaux rejets après la coupe étaient présents dans chaque relevé, de même que Guaiacum sanctum et la cactacée Pilosocereus polygonus, dont étaient présents des individus qui n'avaient pas été coupés (tableau II). Dans les cas de Amyris balsamifera et Capparis ferruginea, présents selon des fréquences moins élevées, comme aussi des succulentes Opuntia sp. et Agave antillarum, il s'agissait aussi d'individus épargnés, tandis que de nouvelles pousses des deux Acacia et de Senna atomaria étaient présentes. Les valeurs d'abondance-dominance selon l'échelle de Braun-Blanquet étaient plus élevées dans le cas des deux cactacées qui n'avaient pas été coupées. Enfin, dans un relevé, un grand tronc de Phyllostylon brasiliense était présent.

\section{Observations}

Dans les endroits où il y avait eu une récolte récente du bois et des traces du charbonnage (ex. : restes de charbon sur le sol), on pouvait observer que les charbonniers n'avaient généralement pas coupé les repousses d'Acacia macracantha de diamètre inférieur à $3 \mathrm{~cm}$, au contraire des tiges plus épaisses de la même espèce. Bien que dans la plupart des cas, les cactacées Pilosocereus polygonus et Opuntia sp. aient été épargnées, on pouvait observer en quelques endroits des souches de Pilosocereus polygonus dont la section indiquait une exploitation récente. Ces souches de $P$. polygonus montraient une lignification avancée dans la zone périphérique des tiges, avec un creux dans le centre. Ces cactus ne sont pas forcément utilisés pour être transformés en charbon, leurs troncs étant localement utilisés comme bords extérieurs des charbonnières (photo 4).

Dans quelques endroits où il y avait des signes d'une exploitation récente, on pouvait observer que quelques arbres de Bursera simaruba de diamètre dépassant $10 \mathrm{~cm}$ n'avaient pas été totalement coupés, mais que leurs troncs principaux avaient été prélevés à une hauteur de 3 à $4 \mathrm{~m}$, tout comme leurs branches. Hors des surfaces d'exploitation récente, on pouvait observer en plusieurs endroits des

Tableau I.

Analyses statistiques des fréquences et des valeurs d'abondance-dominance de 14 espèces (H : Haïti, D : République dominicaine).

\begin{tabular}{|c|c|c|c|c|c|c|c|c|c|c|}
\hline Nom scientifique & $\begin{array}{l}\text { Nom } \\
\text { à Haïti }\end{array}$ & $\begin{array}{l}\text { Nom en } \\
\text { République } \\
\text { dominicaine }\end{array}$ & Famille & Type biologique & $f(H)$ & $f(D)$ & $\mathrm{p}$ & $c-a(H)$ & $c-a(D)$ & $\mathrm{p}$ \\
\hline Cissus trifoliata L. & bwa soldat & vinagrillo & Vitaceae & Liane & 4 & 0 & 0,05 & + & & ns \\
\hline $\begin{array}{l}\text { Senna angustisiliqua (Lam.) } \\
\text { H. S. Irwin \& Barneby }\end{array}$ & cas menaj & carga agua & Caesalpiniaceae & Arbuste & 10 & 0 & 0,00 & + & & 0,00 \\
\hline $\begin{array}{l}\text { Senna atomaria (L.) } \\
\text { H. S. Irwin \& Barneby }\end{array}$ & bwa cabrit & palo de chivo & Caesalpiniaceae & Arbre & 17 & 5 & 0,00 & + & & 0,00 \\
\hline Acacia macracantha Willd. & bayawon & bayahonda prieta & Mimosaceae & Arbre & 17 & 7 & 0,00 & + & & 0,00 \\
\hline $\begin{array}{l}\text { Pilosocereus polygonus (Lam.) } \\
\text { Byles \& G. D. Rowley }\end{array}$ & candelab & cayuco & Cactaceae & Succulente & 18 & 16 & ns & + & & ns \\
\hline Opuntia sp. & raket & tuna & Cactaceae & Succulente & 10 & 9 & ns & + & & ns \\
\hline $\begin{array}{l}\text { Phyllostylon brasiliense } \\
\text { Capanema }\end{array}$ & bwa blan & baitoa & Ulmaceae & Arbre & 15 & 14 & ns & & + & ns \\
\hline Bursera simaruba (L.) Sarg. & gomié & almácigo & Burseraceae & Arbre & 8 & 13 & ns & & + & 0,03 \\
\hline Guaiacum sanctum L. & gaiac & vera & Zygophyllaceae & Arbre & 12 & 18 & 0,01 & & + & 0,00 \\
\hline Acacia scleroxylon Tussac & bwa chandel & candelón & Mimosaceae & Arbre & 5 & 15 & 0,00 & & + & 0,00 \\
\hline Capparis ferruginea L. & oliv & frijolito & Capparaceae & Arbre & 2 & 16 & 0,00 & & + & 0,00 \\
\hline Amyris balsamífera L. & bwa chandelblan & guaconejo & Rutaceae & Arbuste & 5 & 11 & 0,05 & & + & 0,00 \\
\hline Agave antillarum Descourt. & aloés & maguey & Liliaceae & Succulente & 1 & 10 & 0,00 & & + & 0,00 \\
\hline Guaiacum officinale L. & gaiac & guayacán & Zygophyllaceae & Arbre & 1 & 4 & ns & & + & ns \\
\hline
\end{tabular}


individus de $B$. simaruba avec beaucoup de branches à une hauteur de 3 à $4 \mathrm{~m}$, ce qui indique qu'il y avait eu le même type de traitement. Un arbre de cette espèce avait été coupé à une hauteur inférieure à $1 \mathrm{~m}$, et 29 rejets d'un diamètre de 1 à $1,5 \mathrm{~cm}$ avaient été greffés sur le cambium, avec un taux de succès apparent de $97 \%$ (une seule tige greffée était morte, tandis que les autres montraient des signes d'une bonne vitalité), et une longueur de 2 à $3 \mathrm{~m}$.

Les individus jeunes de G. sanctum, de hauteur inférieure à $2 \mathrm{~m}$, et qui généralement gardaient des feuilles vertes lors des relevés, et donc en pleine saison sèche, dans beaucoup de cas n'avaient pas été touchés par les charbonniers. Dans plusieurs endroits où il y avait eu une coupe récente, on pouvait observer une régénération végétative abondante de $P$. brasiliense, avec une longueur des pousses de $10-20 \mathrm{~cm}$.

Des observations complémentaires ont été réalisées. - La technologie appliquée dans la zone pour la construction des charbonnières implique l'utilisation de quantités considérables de terre pour couvrir le bois ramassé et stocké (photo 3). Pour cela, la terre des alentours immédiats des endroits où les charbonnières sont construites est utilisée. Cela signifie que la surface du sol est remuée, ce qui pourrait avoir des conséquences sur la régénération de la végétation. - Dans la zone limitrophe des terrains habités d'Anse-à-Pitre, la structure de la végétation est différente de celle où les relevés ont été réalisés ; on y observe une prédominance des cactus, une présence et un taux de recouvrement relativement plus faibles des espèces qui prédominent dans la forêt sèche du côté haïtien ( $A$. macracantha, S. atomaria), et une absence presque totale de certaines espèces qui sont plus fréquentes du côté dominicain ( $A$. scleroxylon, $A$. balsamifera, C. ferruginea, G. sanctum).
- Aucun incendie n'a été observé dans la zone pendant les mois de février à mai 2015, période qui recouvrait une bonne partie de l'époque sèche. Cela confirme que, dans la zone, le feu n'est pas un facteur écologique majeur, tout au moins actuellement.

- Ponctuellement, des vaches et des chèvres ont été observées dans la forêt sèche du côté haïtien, en de faibles densités (moins de cinq animaux par groupe) et en des points situés à moins de $500 \mathrm{~m}$ des habitations. Du côté dominicain, la présence d'animaux domestiques n'a pas été relevée dans la zone d'étude, bien qu'il y ait des parcours d'animaux dans des endroits plus proches des peuplements humains.

\section{Discussion}

En accord avec la première hypothèse, les paramètres structurels révèlent des contrastes entre les deux côtés de la frontière. Du côté haïtien, on relève un moindre taux de recouvrement des arbres et arbustes, une hauteur moyenne des arbres moins élevée, moins d'arbres de grand diamètre, mais plus de tiges de faible diamètre, plus d'individus de faible taille, et un rapport tiges/pied plus élevé. Ces différences peuvent être facilement attribuées à la coupe des arbres pour la fabrication de charbon de bois du côté haïtien, et à la régénération végétative consécutive de certaines espèces, ceci dans le cas d'une abondance plus grande des diamètres réduits, et des individus multicaules. Ce dernier aspect est en accord avec les résultats de Disla et al. (1986), qui indiquent une bonne capacité de repousser de manière végétative après coupe pour la plupart des espèces d'une forêt sèche près de Santiago de los Caballeros, dans le nordouest de la République dominicaine.

Tableau II.

Espèces présentes dans les six relevés d'exploitation récente du côté haïtien.

Les numéros se réfèrent aux codes d'abondance-dominance de l'échelle de Braun-Blanquet.

\begin{tabular}{|c|c|c|c|c|c|c|}
\hline Espèce & 1 & 2 & 3 & 4 & 5 & 6 \\
\hline Phyllostylon brasiliense & 1 & 1 & 1 & + & + & 1 \\
\hline Guaiacum sanctum & + & 1 & + & + & + & . \\
\hline Pilosocereus polygonus & . & 2 & 2 & + & 2 & + \\
\hline Opuntia sp. & . & 2 & . & . & . & + \\
\hline Senna atomaria & + & . & . & + & + & . \\
\hline Acacia scleroxylon & . & + & + & + & . & . \\
\hline Acacia macracantha & . & . & . & + & . & 1 \\
\hline Amyris balsamifera & . & + & + & . & . & . \\
\hline Capparis ferruginea & 1 & . & . & + & . & . \\
\hline Agave antillarum & . & . & . & + & . & . \\
\hline Croton sp. & + & + & . & . & . & . \\
\hline (non identifié) & . & + & . & + & . & . \\
\hline
\end{tabular}


Les résultats présentés ici sont également en accord avec la seconde hypothèse. Bien que 19 des 28 espèces (68\%) soient présentes dans des relevés des deux côtés de la frontière, il existe dans beaucoup de cas d'importantes différences quantitatives s'agissant des fréquences et des indices d'abondance-dominance.

$\mathrm{Au}$ vu des résultats, la troisième hypothèse selon laquelle les essences préférées pour le bois énergie souffrent davantage de cet impact, et sont de ce fait plus fréquentes du côté de la République dominicaine, doit en revanche être rejetée. Trois des quatre espèces réputées pour la qualité du bois énergie qu'elles fournissent (S. angustisiliqua, S. atomaria, $A$. macracantha) sont plus fréquentes du côté haïtien, tandis que les deux espèces de basse qualité comme combustible ( $B$. simarouba, $P$. brasiliense) le sont davantage du côté dominicain. La fréquence des deux cactacées $P$. polygonus et Opuntia sp., non utilisées comme bois énergie et non consommées par le bétail, est pratiquement la même des deux côtés de la frontière.

Ces différences sont statistiquement significatives. Pour la table de contingence de $2 \times 2$ avec les catégories «bonne qualité comme bois énergie » - « faible qualité comme bois énergie ou neutre », et " plus fréquente en Haïti » - « plus fréquente dans la République dominicaine ou sans différence significative ", le test exact de Fisher indique une association positive $(P>0,02)$ entre la bonne qualité comme bois énergie et une fréquence plus élevée en Haïti. Pour cette analyse, les informations du tableau I et de May (2013) ont été utilisées. Les espèces S. angustisiliqua, $S$. atomaria et $A$. macracantha ont été attribuées à la catégorie « bonne qualité comme bois énergie »- « plus fréquente en Haïti », Acacia scleroxylon à la catégorie «bonne qualité comme bois énergie » - " plus fréquente dans la République dominicaine ou sans différence significative », et toutes les autres espèces représentées dans le tableau I rentrent dans la catégorie "faible qualité comme bois énergie ou neutre » - « plus fréquente dans la République dominicaine ou sans différence significative ». Aucune espèce n'a été attribuée à la catégorie restante, "faible qualité comme bois énergie ou neutre » - « plus fréquente en Haïti ». La liane Cissus trifoliata et la succulente Agave antillarum, espèces présentes dans le tableau I mais qui ne sont pas utilisées comme bois énergie, n’ont pas été considérées dans cette analyse.

Il faut donc supposer que ce n'est pas la préférence comme bois énergie qui détermine les différences dans la composition floristique de la végétation de forêt sèche sur roche calcaire des deux côtés de la frontière de Anse-à-Pitre/ Pedernales, mais qu'existent d'autres facteurs déterminants. Très probablement, ces facteurs sont liés aux perturbations causées par la coupe des arbres avec l'objectif d'utilisation comme bois énergie, et aux caractéristiques biologiques des espèces, comme la capacité de rejeter vigoureusement à partir des souches, et la facilité de colonisation et de dispersion.

Comme la densité des animaux en pâturage libre est relativement faible dans la zone d'étude, hors des périmètres des habitats humains, on peut présumer leur impact limité. Vraisemblablement, les espèces dont la présence et la valeur d'abondance-dominance sont plus élevées du côté haïtien sont favorisées par les animaux. A. macracantha, plus fré-
BOIS ET FOREATS DES TROPIQUES, 2015 , N³26 (4) VÉGÉTATION DE FORÊT SĖCHE / LE POINT SUR.

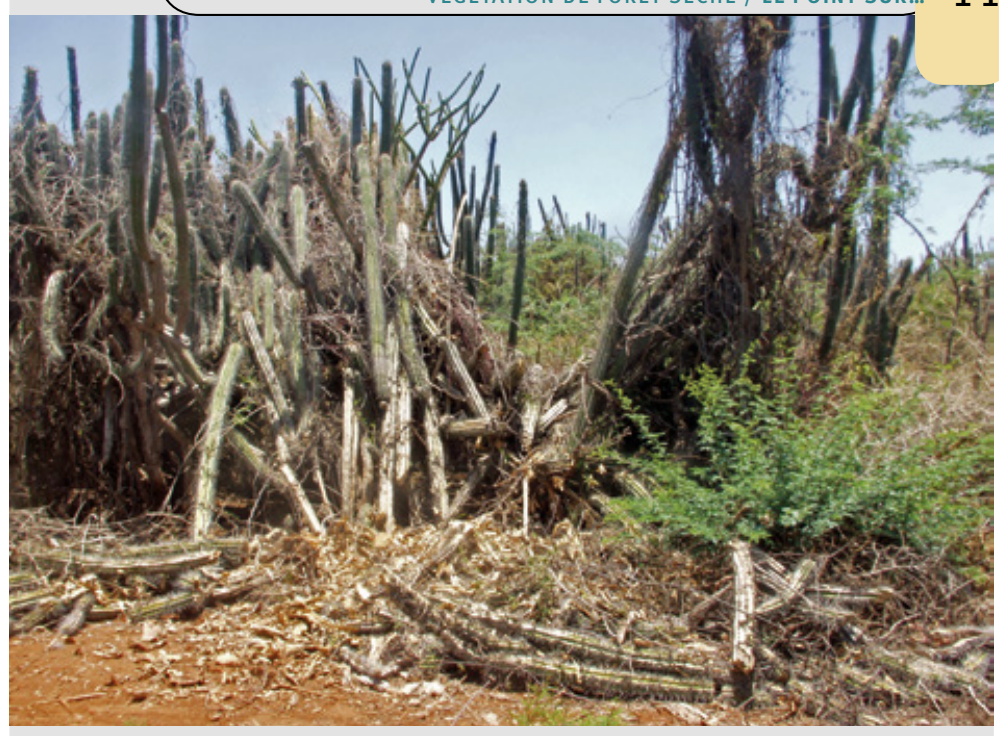

Photo 4.

Forêt sèche près d'Anse-à-Pitre, Haïti. Lieu d'exploitation

récente avec des cactus coupés, probablement pour délimiter les charbonnières.

Photo E. Rupp.

quent du côté haïtien et fortement utilisé pour le charbonnage, est une bonne espèce colonisatrice, dispersée de manière endozoochore par les vaches (Howard et Zanoni, 1989) et, selon les informations des interlocuteurs locaux, également par les chèvres. Le même processus pourrait intervenir pour S. atomaria, dont le nom vernaculaire, tant haïtien que dominicain (tableau I), indique une préférence par les chèvres, qui mangent tant les fruits verts que les fruits secs contenant des semences mûres, selon un interlocuteur dominicain d'un village voisin de la frontière. S'il y a une sélection des espèces via le pâturage des animaux de parcours, actuellement beaucoup plus important du côté haïtien que du côté dominicain, celle-ci tend à favoriser ces deux espèces de bonne qualité comme bois de feu, en contribuant à leur dispersion, beaucoup plus qu'à favoriser des espèces qui ne sont pas consommées, comme les deux cactacées mentionnées plus haut. La seule espèce qui est plus fréquente du côté dominicain et dont le feuillage est d'une bonne qualité fourragère reconnue (Geilfus, 1994) est Bursera simarouba. Quant à la structure de la végétation, la densité plus faible des individus de taille au-dessous de $0,5 \mathrm{~m}$ du côté haïtien, au contraire des classes de hauteur entre 0,5 et $5 \mathrm{~m}$, pourrait être due à l'impact des animaux de parcours, soit par dommage mécanique, soit par consommation (figure 2). Il est également possible que cette différence de densité résulte de la régénération de tiges par voie végétative, plus forte du côté haïtien.

Il est connu que la capacité de régénération végétative d'A. scleroxylon, la seule des espèces préférées comme bois énergie qui est moins fréquente dans les relevés du côté haïtien que dans les relevés du côté dominicain, est bonne (Disla et al., 1986), mais il n'existe pas à notre connaissance d'information sur les caractéristiques de colonisation de cette espèce. En tout cas, son caractère d'espèce endémique de La Hispaniola (May, 2001) pourrait indiquer que sa capacité à coloniser des terrains perturbés est plus limitée que pour $A$. macracantha. Étant donné que, pour obte- 


\section{Recommandations}

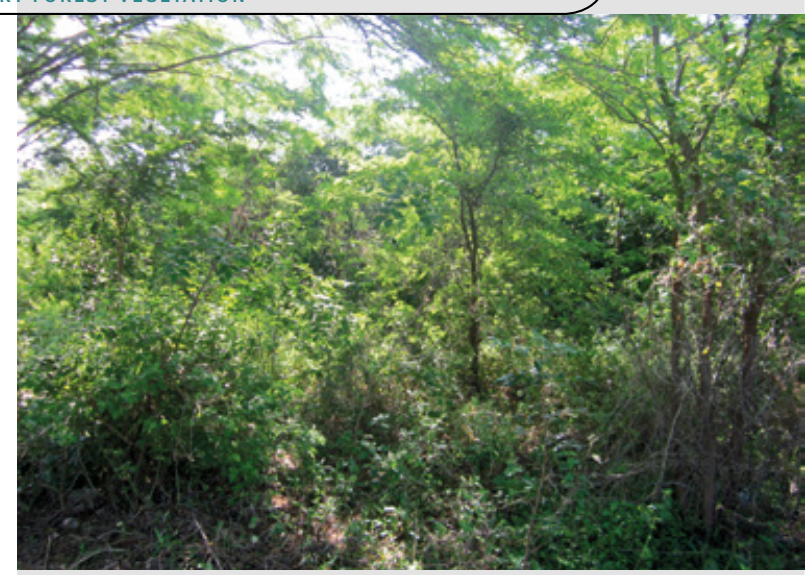

Photo 5.

Forêt sèche près de Pedernales, du côté dominicain, avec les espèces Acacia macracantha et Phyllostylon brasiliense dans le fond.

Photo E. Rupp.

nir la terre pour la construction des charbonnières, il faut remuer la surface du sol, une faible capacité de colonisation doit être un handicap dans ces conditions.

Les trois espèces plus fréquentes du côté haïtien sont des légumineuses, tandis que dans le groupe d'espèces plus fréquentes du côté dominicain ne se rencontre qu'une seule légumineuse (Acacia scleroxylon). La tendance selon laquelle, dans les situations de colonisation des forêts sèches, prédominent des légumineuses, a été également relevée par Romero-Duque et al. (2007) et ZamoraCrescencio et al. (2011) au Mexique, par Aguirre Mendoza et al. (2013) en Équateur, par Roth (1999) dans la zone de forêt sèche du nord-ouest de la République dominicaine ou encore par Badji et al. (2014) au Sénégal.

Il se manifeste une sélection des bois à couper de la part des charbonniers, comme la préservation des repousses quand les diamètres sont très faibles, même pour une espèce comme $A$. macracantha, en principe préférée pour la production de charbon. Dans les cas de $B$. simarouba et $P$. brasiliense, la faible qualité comme bois énergie est en concordance avec le fait qu'elles sont souvent épargnées en raison de leur mauvaise qualité comme bois de feu ou pour la production de charbon, bien que cela ne soit pas toujours le cas comme en témoignent les boutures abondantes de $P$. brasiliense dans des endroits de coupe récente. Étant donné l'importance de l'élevage des ruminants dans la zone, l'utilité des feuilles de $B$. simarouba comme aliment pour les chèvres (Geilfus, 1994) pourrait aussi jouer un rôle pour que cette espèce soit préservée en quelques endroits. En tout cas, autant pour $B$. simarouba que pour $P$. brasiliense, la sélection négative par les charbonniers ne se traduit pas par des fréquences plus élevées du côté haïtien, bien que la capacité de repousser au ras du sol après la coupe de $P$. brasiliense soit excellente.

Enfin, l'intensité de l'utilisation joue certainement un rôle. L'état de la végétation dans la proximité de la zone habitée d'Anse-à-Pitre, qui présente moins d'abondance-dominance de certaines espèces qui sont fréquentes dans les relevés du côté haïtien, comme $A$. macracantha et $S$. atomaria, mais aussi une prédominance des cactacées et une structure arbustive, est certainement dû à une utilisation très intense comme bois énergie, et probablement aussi comme fourrage.
En Haïti, la demande de charbon comme source énergétique, l'état de pauvreté d'une partie importante de la population, de même que le manque d'alternatives actuelles, ne laissent d'autre choix à beaucoup de personnes que de rejoindre la filière du charbon comme producteurs ou consommateurs, malgré le fait qu'elles soient conscientes de l'impact des prélèvements de bois. À cause de la situation de pauvreté, qui conduit à prioriser les solutions à court terme, il n'est pas facile de concevoir des mesures pour améliorer l'utilisation des ressources qui soient acceptables pour les habitants ruraux et mises en pratique. Au lieu de la stigmatisation des paysans haïtiens comme agents de la destruction environnementale, et de la prohibition de l'utilisation du bois énergie, irréaliste dans les conditions sociales et économiques actuelles, il serait plus constructif de développer et promouvoir des méthodes et techniques pour rendre plus durable la production du charbon, en prenant en compte toute la filière jusqu'à la consommation, avec la participation des paysans producteurs.

Dans certaines conditions, des plantations pourraient être réalisées dans un objectif de production de bois énergie. Dans la zone de l'étude, il semble toutefois plus judicieux de développer une bonne gestion des ressources existantes. La bonne résilience des espèces qui fournissent du bois énergie de bonne qualité, comme les légumineuses A. macracantha et $S$. atomaria, offre un potentiel en faveur d'une gestion plus durable des ressources de bois énergie. Il faudrait réaliser des essais de terrain pour connaître plus sûrement les perspectives d'appliquer des techniques de traitement des repousses, en laissant seulement deux ou trois tiges, dont l'accroissement serait alors plus vigoureux. Un traitement de ce type pourrait favoriser le retour à une structure forestière, avec des arbres de plus haute taille, un couvert plus fourni et une productivité plus élevée. Cette mesure devrait être accompagnée d'une utilisation selon des cycles de rotation d'une durée adéquate. La durée serait un compromis entre les nécessités des producteurs à court terme et les nécessités de régénération de la végétation. Cependant, des mesures comme celle décrite ici, avec l'objectif d'une gestion durable des ressources de bois énergie, ne garantiraient pas forcément la restauration des peuplements d'autres espèces de la forêt sèche, importantes pour la biodiversité, comme A. scleroxylon, Amyris balsamifera, $C$. ferruginea et $G$. sanctum, dont les populations sont affectées négativement par l'utilisation actuelle de la forêt.

\section{Remerciements}

L'auteur remercie Masani Accimé (ONG Iguana Foundation, Anse-à-Pitre, Haïti), Ernst Rupp (Grupo Jaragua, Oviedo, République dominicaine) et l'agronome Étienne Hérard qui l'avait accompagné dans le premier voyage de reconnaissance dans la forêt sèche près d'Anse-à-Pitre, pour des intéressants échanges d'idées sur la problématique de la forêt sèche, de la production du charbon de bois et des possibles formes alternatives d'utilisation dans la zone de la frontière dominico-haïtienne. 


\section{Références bibliographiques}

Aguirre Mendoza X., Betancourt Figueras Y., Geada López G., 2013. Regeneración natural en los bosques secos de la provincia de Loja y utilidad para el manejo local. Revista CEDAMAZ, 3 (1): 54-65.

Artana D., Auguste S., Bour J. L., Navajas F., Panadeiros M., Guzmán R. M., 2006. El gasto público en República Dominicana. Re2-06-011, Serie de estudios económicos y sociales. Washington, DC, USA, Banco Interamericano de Desarrollo.

Badji M., Sanogo D., Akpo L. E., 2014. Dynamique de la végétation ligneuse des espaces sylvo-pastoraux villageois mis en défens dans le Sud du Bassin arachidier au Sénégal. Bois et Forêts des Tropiques, 319 (1) : 43-52. [En ligne] http://bft.cirad.fr/cd/BFT 319 43-52.pdf

Boccanfuso D., Siméon A., 2006. Dynamique de la pauvreté en Haïti et ses déterminants. Université de Sherbrooke, GRÉDI (Groupe de recherche en économie et développement international), Canada. Cahier de recherche, 06/15.

Díaz R., 2003. Diagnóstico del sector forestal en la R. D. Santo Domingo, République dominicaine, Cámara Forestal Dominicana/GTZ, 68 p.

Disla M. T., Gómez A. R., Mercedes J., 1986. Rebrotes en tala rasa de bosque seco. Santiago de los Caballeros, République dominicaine, Instituto Superior de Agricultura, Nota técnica, 31, $19 \mathrm{p}$.

Geilfus F., 1994. El árbol al servicio del agricultor. Manual agroforestal para el desarrollo rural. 2. Guía de especies. Turrialba, Costa Rica, EndaCaribe, CATIE, 778 p.

Hartshorn G., Antonini G., Dubois R., Harcharik D., Heckadon S., Newton A., Quesada C., Shores J., Staples G., 1981. La República Dominicana. Perfil ambiental del país. Un estudio de campo. Mc Lean, VA, USA, JRB Associates, 134 p.

Hofstad O., 1997. Woodland deforestation by charcoal supply to Dar es Salaam. Journal of Environmental Economics and Management, 33 (1): 17-32.

Hountondji Y. C., Gaoué O. G., Skopon N., Ozer P., 2013. Analyse écogéographique de la fragmentation du couvert végétal au nord Bénin : paramètres dendrométriques et phytoécologiques comme indicateurs in situ de la dégradation des peuplements ligneux. Géo-Eco-Trop., 37 (1 ) : 53-70.

Howard R. A., Zanoni T. A., 1989. Two atypical examples of seed distribution in the Dominican Republic. Moscosoa, 5: 216-225.

Mama A., Bamba I., Sinsin B., Bogaert J., De Cannière C., 2014. Déforestation, savanisation et développement agricole des paysages de savanes-forêts dans la zone soudano-guinéenne du Bénin. Bois et Forêts des Tropiques, 322 (4) : 6575. [En ligne] http://bft.cirad.fr/cd/BFT 322 65-75.pdf

May T., 2001. El endemismo de plantas vasculares en la República Dominicana, en relación con condiciones ambientales y factores biogeográficos. Moscosoa, 12: 60-78.
May T., 2013. Plantas preferidas para leña en la zona de bosque seco de Pedro Santana y Bánica, República Dominicana. Aspectos etnobotánicos y de manejo sustentable. Ambiente y Desarrollo, 17 (33): 71-85.

Molina Colón S., Lugo A. E., 2006. Recovery of a subtropical dry forest after abandonment of different land uses. Biotropica, 38 (3): 354-364.

Murphy P. G., Lugo A. E., 1986. Ecology of tropical dry forest. Annual Review of Ecology and Systematics, 17: 67-88.

Mwampamba T. H., 2007. Has the woodfuel crisis returned? Urban charcoal consumption in Tanzania and its implications to present and future forest availability. Energy Policy, 35 (8): 4221-4234.

Peltier R., Dubiez E., Diowo S., Gigaud M., Marien J.-N., Marquant B., Péroches A., Proces A., Vermeulen C., 2014. Assisted natural regeneration in slash-and-burn agriculture: results in the Democaratic Republic of Congo. Bois et Forêts des Tropiques, 321 (3): 67-79. [En ligne] http://bft.cirad.fr/ cd/BFT 321 67-79.pdf

Racicot A., 2011. Durabilité des combustibles de substitution au bois énergie en Haïti - Filières renouvelables pour la cuisson des aliments. Thèse, Sherbrooke University, Québec, Canada, 99 p.

Romero-Duque L. P., Jaramillo V., Pérez-Jiménez A., 2007. Structure and diversity of secondary dry forests in Mexico, differing in their prior land-use history. Forest Ecology and Management, 253: 38-47.

Roth L. 1999. Anthropogenic change in subtropical dry forest during a century of settlement in Jaiquí Picado, Santiago province, Dominican Republic. Journal of Biogeography, 26: 739-759.

SEIC/DGM, 1991. Mapa geológico de la República Dominicana. Santo Domingo, République dominicaine.

Tabuti J. R. S., Dhillion S. K. A., Lye K. A., 2003. Firewood use in Bulamogi County, Uganda: species selection, harvesting and consumption patterns. Biomass and Bioenergy, 25: 581-596.

Université de Louvain, 1998. Étude sur la déforestation et la dégradation de l'environnement à Haïti. In: FAO (éd.). Forestry policies in the Caribbean. Volume 2: Reports of 28 selected countries and territories. Rome, Italie, FAO, FAO Forestry Papers 137 (2): 323-355.

Zamora-Crescencio P., Domínguez-Carrasco M. R., Villegas P., Gutiérrez-Báez C., Manzanero-Azevedo L. A. et al., 2011. Composición florística y estructura de la vegetación secundaria en el norte del Estado de Campeche, México. Boletín de la Sociedad Botánica de México, 89 : 27-35. 